\title{
Digital Atlases of the Antennal Lobe in Two Species of Tobacco Budworm Moths, the Oriental Helicoverpa assulta (Male) and the American Heliothis virescens (Male and Female)
}

\author{
BENTE G. BERG,${ }^{1,2 *}$ C. GIOVANNI GALIZIA, ${ }^{3}$ ROBERT BRANDT, ${ }^{3}$ \\ AND HANNA MUSTAPARTA ${ }^{1}$ \\ ${ }^{1}$ Department of Zoology, Norwegian University of Science and Technology, \\ N-7491 Trondheim, Norway \\ ${ }^{2}$ Department of Psychology, Norwegian University of Science and Technology, \\ N-7491 Trondheim, Norway \\ ${ }^{3}$ Institut für Biologie-Neurobiologie, Freie Universität Berlin, D-14195 Berlin, Germany
}

\begin{abstract}
The antennal lobe of the moth brain is the primary olfactory center processing information about pheromones and plant odors. We present here a digital atlas of the glomerular antennal lobe structures in the male of Helicoverpa assulta and the male and female of Heliothis virescens, based on synaptic antibody staining combined with confocal microscopy. The numbers of the glomeruli in the three specimens were similar, 65, 66, and 62, respectively. Whereas the male antennal lobe has a macroglomerular complex consisting of three and four units in the two species, the female lobe has two enlarged glomeruli at a corresponding position, near the entrance of the antennal nerve. Another large glomerulus, showing homology in the three specimens, is ventrally located. The small size of the heliothine moths is advantageous for confocal microscopy because the entire brain can be visualized as a single image stack. The maps are freely accessible on the internet, and the digital form of the data allows each atlas to be rotated and sectioned at any angle, providing for the identification of glomeruli in different preparations. J. Comp. Neurol. 446:123-134, 2002. ๑ 2002 Wiley-Liss, Inc.
\end{abstract}

Indexing terms: olfactory system; glomeruli; heliothine moths; anatomical reconstruction; confocal microscopy; internet

Olfactory detection in insects is performed by receptor neurons housed in sensilla, most of which are located on the antennae. The sensory neurons send projections directly to the antennal lobe, the primary olfactory center of the insect brain. Here, the terminals make synapses with second-order neurons in spheroidal structures called glomeruli (Rospars, 1988; Boeckh and Tolbert, 1993). Shepherd (1974) defines a glomerulus as "a synaptic complex enclosed in glial membranes or otherwise set apart." This definition applies to most animal species, vertebrates as well as invertebrates. In some insects, such as lepidopterans, the males possess a specific macroglomerular complex (MGC) in the antennal lobe, forming a first relay for processing information about sexual pheromones and interspecific signals. The functional significance of the individual MGC compartments has been thoroughly investi- gated in several moth species by functional tracing of the pheromone receptor neurons as well as of the antennal lobe projection neurons (Christensen et al., 1991, 1995;

Grant sponsor: The Norwegian Research Council; Grant number: 125821/410; Grant number: 133958/420; Grant sponsor: German Research Ministry BMBF; Grant number: 0310961; Grant sponsor: Volkswagenstiftung; Grant number: 175/399.

*Correspondence to: Bente Gunnveig Berg, Department of Psychology, Norwegian University of Science and Technology, N-7491 Trondheim, Norway. E-mail: bente.berg@svt.ntnu.no 
Hansson et al., 1991; Vickers et al., 1998; Berg et al., 1998).

All heliothine species so far investigated use a multicomponent pheromone blend containing the same chemicals in different compositions. The receptor neurons detecting the "insect-produced" compounds in the various species can be classified into types according to their specificities, each type receiving information about one chemical (Mustaparta, 1997). Because of the detailed knowledge of physiology and behavior involved in intra- as well as interspecific pheromone communication, the MGC in heliothine moths also offers interesting comparative aspects concerning functional organization of the glomerular units. In the male of the American tobacco budworm moth Heliothis virescens, the number of identified receptor neuron types detecting insect-produced substances matches perfectly the number of glomerular units constituting the MGC. Four behaviorally relevant chemicals are detected by four specific receptor neuron types, each projecting in one of four MGC compartments (Hansson et al., 1995; Berg et al., 1998). The two largest units receive input from neurons carrying information used in behavioral attraction and the two smallest from neurons responsible for interruption of attraction. Furthermore, there appears to be a correspondence between the input and the output information of each MGC compartment, supporting the idea that the individual glomeruli are particularly involved in processing information about one of the chemicals (Christensen et al., 1995; Vickers et al., 1998; Berg et al., 1998).

The role of the ordinary glomeruli, which are the targets for the receptor neurons detecting plant odor volatiles, has until now remained relatively unexplored. In contrast to the pheromone system, offering a small number of easily recognizable MGC compartments, the ordinary glomeruli are numerous and very challenging for identification. Another important task is to identify relevant odorants for which the plant odor receptor neurons have evolved. Recently, a major receptor neuron type responding with high sensitivity and selectivity to the sesquiterpene germacrene $\mathrm{D}$ has been found in the $H$. virescens female (Røstelien et al., 2000). The large number of these neurons indicates the significance of the odorant for the detection of plants by female moths. A functionally similar receptor neuron type has also been identified in the closely related species $H$. assulta. An interesting question is whether the axons of all germacrene $D$ receptor neurons project to a single glomerulus and, if so, whether this particular glomerulus can be considered as homologous in different species of heliothine moths.

In the present study, we have mapped the individual glomeruli of the antennal lobes in two heliothine species, the male and female of $H$. virescens and the male of $H$. assulta. As a contribution in a series of digital atlases of the insect antennal lobe that are published on the internet, including the antennal lobe of the honeybee Apis mellifera (Galizia et al., 1999) and the fruit fly Drosophila melanogaster (Laissue et al., 1999), we present here the antennal lobes of two moth species. The internet publication of the present data makes these atlases freely available as a tool, allowing other researchers to map physiological responses to morphologically identified glomeruli. The results obtained from studies of the pheromone system in combination with the recent data on the plant odor system make heliothine moths suitable model organisms for investigating the function of individual glomeruli as well as enlightening similarities and differences between related species.

\section{MATERIALS AND METHODS Antibody staining procedure}

$H$. virescens and $H$. assulta pupae, originating from laboratory cultures, were kindly provided by Dr. Max Angst (Novartis Crop Protection, Basel, Switzerland), Dr. K.S. Boo (Seoul National University, Suwon, Republic of Korea), and Dr. Sadahiro Tatzuki (The University of Tokyo, Tokyo, Japan). Newly eclosed insects were used for the experiments. The brains were dissected from the head and kept overnight in a $4 \%$ paraformaldehyde fixation at $4^{\circ} \mathrm{C}$. After fixation, the tissue was rinsed $(10 \times 1$ hour $)$ with $0.1 \mathrm{M}$ phosphate-buffered saline (PBS), $\mathrm{pH} 7.4$, with added 1\% Triton X-100 (PBST: $8 \mathrm{~g} \mathrm{NaCl}, 0.2 \mathrm{~g} \mathrm{KCl}, 1.44 \mathrm{~g}$ $\mathrm{Na}_{2} \mathrm{HPO}_{4}, 0.24 \mathrm{~g} \mathrm{KH}_{2} \mathrm{PO}_{4}, 1 \mathrm{ml}$ Triton). The brains were preincubated in PBST, $\mathrm{pH} 7.4,+10 \%$ normal goat serum (NGS; Sigma) for 2 hours and then incubated for 60 hours at room temperature with the primary antisera SYNORF1 and nc46, each diluted 1:30 in 10\% NGS. The SYNORF1 detects a particular part of the synapsin molecule, whereas the antigen of nc46 is not known. The mixture of the two instead of one is shown to be favorable in honeybees. Both primary antibodies stem from a screen of Drosophila synapse protein (Reichmuth et al., 1995; Klagges et al., 1996) and were kindly provided by Dr. E. Buchner (Würzburg, Germany). Only one type of secondary antibody was used. After incubation in the primary antisera, the brains were rinsed $(10 \times 1$ hour $)$ in PBST and then incubated in the secondary antiserum, a Cy3-conjugated mouse anti-rabbit antibody (Jackson Immunoresearch, West Grove, PA; diluted 1:200 in 10\% NGS), and kept in darkness for ca. 36 hours at room temperature. After 10 rinses, each for 1 hour, in PBS (in the darkness), the brains were dehydrated through a graded series of alcohols $(50 \%, 70 \%, 90 \%, 99 \%, 2 \times 100 \%$, each for $10 \mathrm{~min})$ and cleared in methyl salicylate. The brains were oriented in a frontal position and embedded in Permount (Sigma, St. Louis, MO) in double-sided custom slides.

\section{Confocal laser scanning microscopy}

Twenty-eight brains were reconstructed by laser scanning microscopy (LSM). For further analyses, six were selected (two $H$. assulta males, two $H$. virescens males and two $H$. virescens females). One of each of three preparations (all named specimen 1) was used as a model to create the digital atlases. The other three (all named specimen 2) were studied for comparing the number and position of the glomeruli between individuals of each type.

The three brains (specimens 1) used for the creation of the atlases were analyzed with a Leica TCS 4D confocal microscope (DM RBE). Dry immersion objectives were used to scan the specimens, a Leica HC PL APO $10 \times / 0.4$ dry objective for the whole brain and a Leica PL APO $20 \times / 0.6$ for the antennal lobe. The red-orange fluorescent Cy3 was excited by the $568 \mathrm{~nm}$ spectral line of an $\mathrm{Ar}-\mathrm{Kr}$ $150 \mathrm{~mW}$ mixed gas laser and detected with a longpass filter (LP590). The brains were scanned in a set of frontal planes. To remove random noise, a scan average of eight lines was applied. The spatial sampling frequency was $0.72 \mu \mathrm{m} /$ voxel in the image plane $(512 \times 512$ pixels $)$ and 
$2.01 \mu \mathrm{m} /$ voxel in the axial direction. The entire stack of images contained 130 sections for the $H$. assulta brain specimen, 150 for the $H$. virescens male, and 160 for the $H$. virescens female. The data were stored on a CD-ROM. The $\mathrm{z}$-axis dimension was corrected by a factor of 1.6 to allow for a z-axis shortening introduced by the refractive index mismatch in the optical pathway when using a dry objective. Under these conditions, movement of the microscope stage by $1.25 \mu \mathrm{m}$ per slice corresponded to an optical interslice distance of $2 \mu \mathrm{m}$.

The three brains (specimens 2) used for comparison of the glomerular pattern were analyzed with a Zeiss LSM 510 LSM. A dry immersion objective, plan-Neofluar $20 \times 1$ 0.5 , was used to scan the preparations. An excitation wavelength of $543 \mathrm{~nm}$ was used with a He-Ne $1 \mathrm{~mW}$ mixed-gas laser. The spatial sampling frequency was similar to that described above, $0.9 \mu \mathrm{m} /$ voxel in the image plane $(512 \times 512$ pixels $)$ and $1.9 \mu \mathrm{m} /$ voxel in the axial direction.

\section{Three-dimensional reconstructions and data analyses}

The choice of the six scanned preparations used for further analyses was based on the quality of the stainings. The selected brains displayed a satisfactory extent of fluorescence staining in each section of the entire image stack, making it possible to discriminate the glomerular structures from the background throughout the antennal lobe.

To analyze the raw data, professional, general-purpose visualization and $3 \mathrm{D}$ reconstruction software were used: Amira 2.1 (Indeed GmbH, Berlin; http://www.amiravis. com) and Zeiss 3D for LSM 510 2.8. The digital atlases were created in Amira, based on the extraction of polygonal surface models from 3D image data by tracing the individual glomeruli section by section. The segmentations were made in frontally oriented optical slices. Each digital reconstruction was based on optical slices of one specimen (specimen 1). Different tools were used for the segmentation analysis. By adjusting the gray value mapping in each slice, the anatomical structures were discriminable from the background. The simultaneous display of the data sets in the xy-, xz-, and yz-planes made it easier to decide the outlines in the z-direction. For the H. assulta male and the $H$. virescens female preparation, each image section of the entire stack was analyzed separately; i.e., outlines of the glomeruli were traced every second micrometer. For the $H$. virescens male, two image sections were put together, so that tracings were made every fourth micrometer. To visualize the raw data from the confocal microscope in $3 \mathrm{D}$, as shown in Figure 1, the "volume rendering" tool of Amira was used. This technique, which gives a $3 \mathrm{D}$ impression of the whole data set without segmentation, is based on the attribution of light emission and absorption to every voxel of the volume as a function of its staining intensity, which is subsequently projected onto the viewing plane.

For comparing the glomerular patterns in two individuals of each type, a second reconstruction of one $H$. assulta male, one $H$. virescens male, and one $H$. virescens female was made using the software program Zeiss 3D for LSM 510 2.8. In $H$. assulta a comparison of the glomerular pattern was carried out by identifying each glomerulus in specimen 2 according to specimen 1 using the two stacks of sequential optical images. In $H$. virescens, 36 glomeruli in the male specimen 2 and 32 glomeruli in the female specimen 2 were identified by comparing the respective $3 \mathrm{D}$ reconstructions of the raw data with the corresponding atlases. In addition, the total number of glomeruli was counted by analyzing the stacks of sequential optical images in the two sexes. To visualize the raw data of each specimen 2 in 3D, the "projection" tool of LSM 510 was used. This function calculates a series of sections after rotating the data package about one of three axes. The final figures were edited with Adobe Pagemaker 6.5. Some of the images were adjusted for brightness and contrast with Adobe Photoshop v. 5.5.

\section{Terminology}

The MGC structures in the two male species and the enlarged glomeruli in the female (LFG), positioned at the entrance of the antennal nerve, were easy to recognize. These glomeruli were given names according to previous descriptions for heliothine moths and the sphinx moth Manduca sexta (Christensen et al., 1991; Berg et al., 1998, 1999; King et al., 2000). The remaining ordinary glomeruli were given numbers for identification, roughly following a system in which each glomerulus was given a value according to its position along the $z$-axis, so that frontally located glomeruli have low numbers, whereas those located posteriorly have high numbers. The landmarks used for orientation of the antennal lobe were the antennal nerve and the two cell soma clusters, the lateral and the medial. These brain structures were included in the atlases for orientation purposes.

\section{RESULTS Histology}

The neuropil-specific monoclonal antibody staining gave rise to an intense labeling of the glomeruli, revealing them as anatomically separable units (Fig. 1A,B). The total assembly of glomeruli forms the outer part of the spheroidal antennal lobe, with the central area devoid of any glomerulus (Fig. 2). The antennal lobes are the most anterior and prominent part of the deutocerebrum. The remaining deutocerebrum, the antennal mechanosensory and motor center, which was also strongly labeled, showed no glomerular organization (Fig. 1A,B). With this technique we also obtained clear labeling of the protocerebral structures, the calyces of the mushroom bodies, located posteriorly in the moth brain, as well as of the subesophageal ganglion and the internal structures of the optic lobes (Fig. 1B,C). Weak staining of the antennal nerve appeared. However, no nerve tracts within the antennal lobe were visible. The labeling of the two main cell clusters, the lateral and the medial, clearly appeared in all preparations.

Comparison of the six analyzed antennal lobes showed minor differences in size. The maximal thickness, i.e., in the anterior-posterior direction ( $z$-axis) of the antennal lobe of specimen 1 was $216 \mu \mathrm{m}, 236 \mu \mathrm{m}$, and $264 \mu \mathrm{m}$ for $H$. assulta male and $H$. virescens male and female, respectively, whereas specimen 2 measured $225 \mu \mathrm{m}, 238 \mu \mathrm{m}$, and $228 \mu \mathrm{m}$, respectively. The high resolution of the raw data allowed individual delineation of and numbering of the glomeruli, as shown in Figures 2 and 3. 

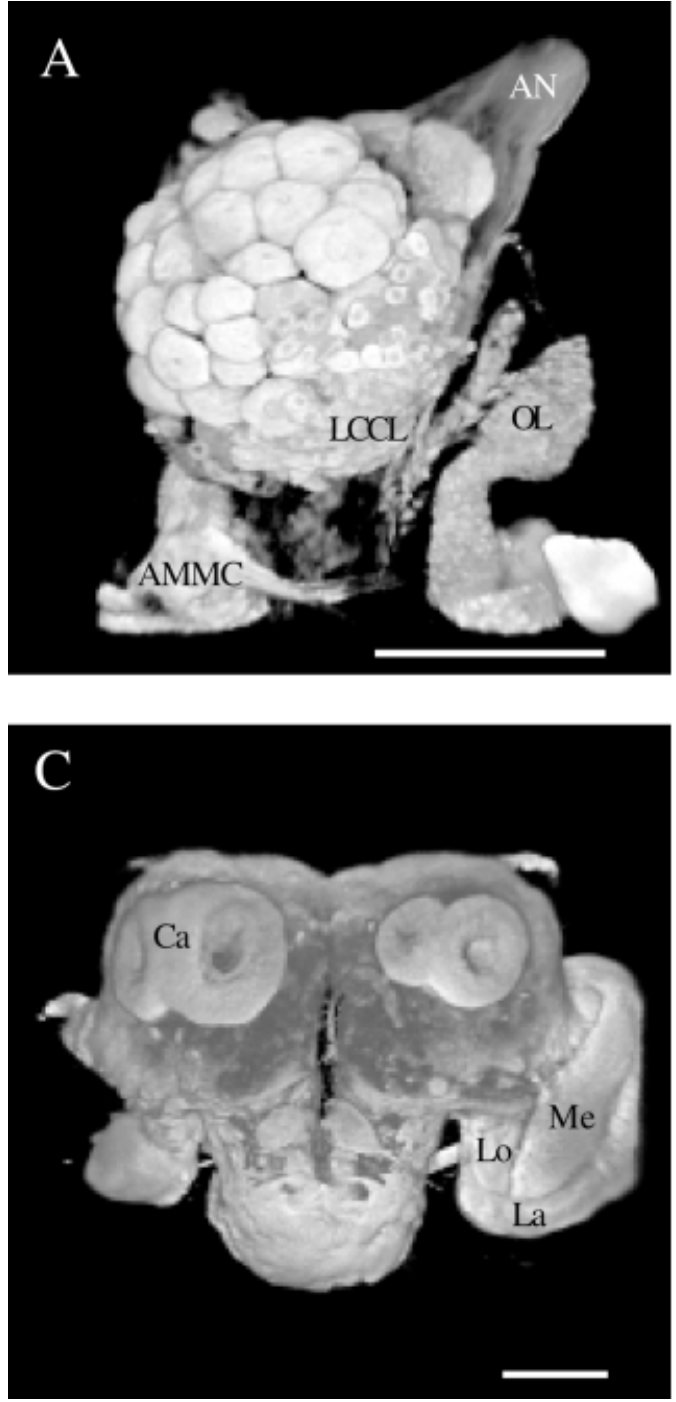

Fig. 1. Three-dimensional confocal reconstructions of the moth brain (Helicoverpa assulta male). A: Deutocerebrum of the left brain hemisphere, consisting of the two main subdivisions; the antennal lobe (AL) and the antennal mechanosensory and motor center (AMMC). The glomerular structure, characteristic of the primary olfactory center, clearly appears. B: Whole brain in a frontal view.

\section{Reconstructions of the glomeruli in the H. assulta male}

$\boldsymbol{M G C}$. Three glomeruli located dorsolaterally in the antennal lobe, clearly separated from the array of ordinary glomeruli, form the MGC of the $H$. assulta male (Figs. 2B-D, 3B-D, 4C,D, 5A,D-F). They have previously been shown to receive information about "insectproduced" signals (Berg et al., 1999). The largest MGC unit, the cumulus, is positioned at the center of the antennal nerve entrance, between the dorsomedial and the ventral compartments. In following the optical sections in the direction of the $z$-axis from anterior to posterior, the cumulus appeared first, then the dorsomedial compartment, and finally the ventral compartment. The cumulus, appearing in frontal sections as a cone-shaped structure,

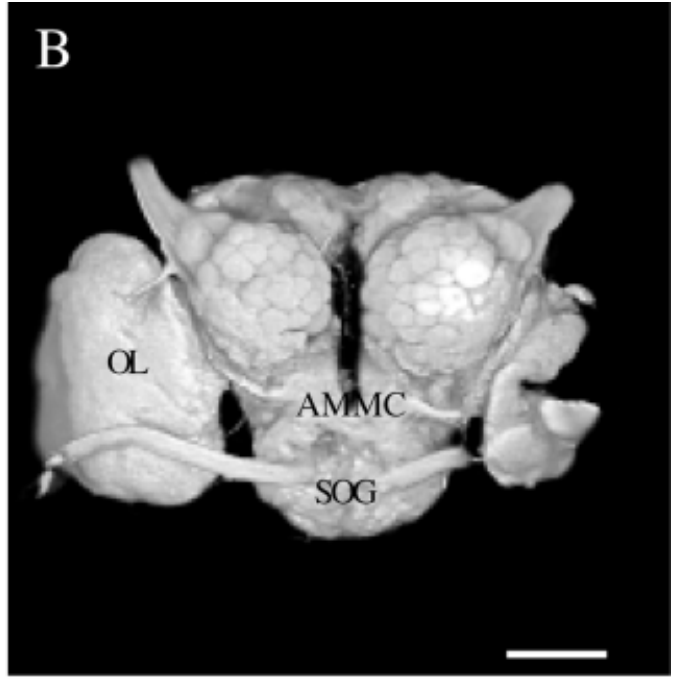

C: Whole brain viewed from behind. The calyces $(\mathrm{Ca})$ of the mushroom bodies clearly appear as prominent structures of the protocerebrum. The right optic lobe (OL) with the lamina (La), medulla (M), and lobula (L) is also shown. LCCl, lateral cell cluster; SOG, subesophageal ganglion; AN, antennal nerve. Scale bars $=200 \mu \mathrm{m}$.

measured $92 \mu \mathrm{m}$ in maximal thickness in specimen 1 , whereas the dorsomedial and the ventral compartments were 78 and $84 \mu \mathrm{m}$, respectively. The maximal thickness measured in each of the three MGC compartments of specimen 2 was similar (Table 1). The position of the MGC was about $50 \mu \mathrm{m}$ posterior to the most anterior glomerulus.

Ordinary glomeruli. Sixty-two ordinary glomeruli were found in the male of $H$. assulta (Figs. 2, 3). Most of them were spheroid or slightly oblong, with a diameter in the range of $40-60 \mu \mathrm{m}$. One glomerulus particularly large in size, G52, was recognized as the so-called labial pit organ (LPO) glomerulus (Figs. 2C-F, 3D,E, 4D, 5C,D,G), elaborately described for $M$. sexta on the basis of its position as the most ventral in the antennal lobe (Kent et al., 

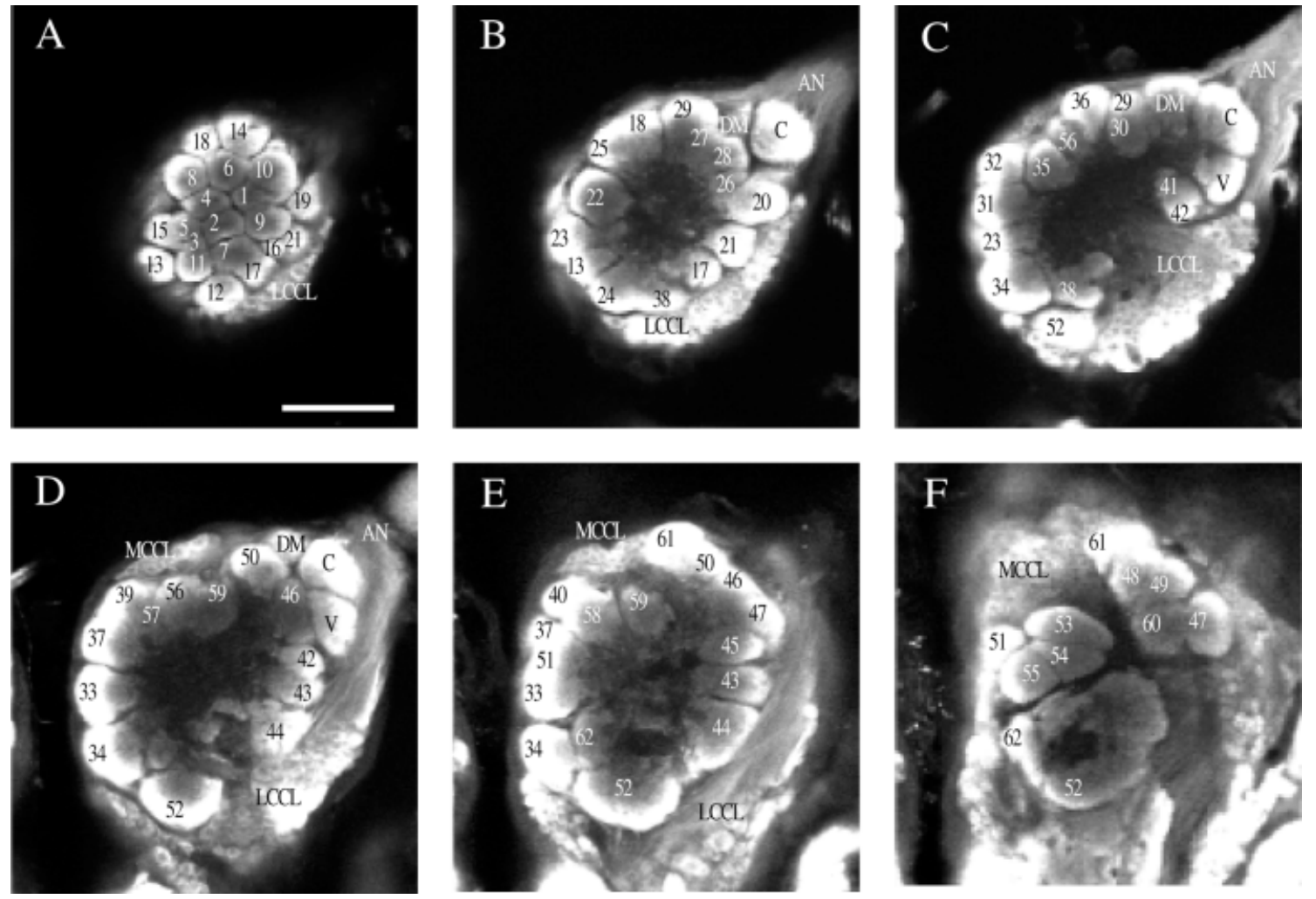

Fig. 2. Helicoverpa assulta male (specimen 1). Images of the antennal lobe in six different planes, showing the 65 glomeruli and the two main cell clusters. Each glomerulus appeared in 20-30 sequential images, making it possible to delineate them, although some sections displayed diffuse borderlines, such as the borders of G33, G37, and G51 in E. A: At $40 \mu \mathrm{m}$ depth, the most anterior glomeruli are seen, including the small unit G16. B: At $68 \mu \mathrm{m}$ depth, two of the three MGC units [the cumulus (C) and the dorsomedial compartment (DM)] appear, residing outside the array of ordinary glomeruli. C: At $100 \mu \mathrm{m}$ depth, all three MGC compartments [C, DM, and the ventral (V)] are visible. Both groups of cell bodies are present; the large lateral cell cluster (LCCl) and the smaller medial cell cluster (MCCl). D: At 132 $\mu \mathrm{m}$ depth, the LPO glomerulus (G52) can be seen with an adjacent large glomerulus (G34). E: At $164 \mu \mathrm{m}$ depth, the ordinary glomerulus, G61, positioned behind the dorsomedial MGC compartment distinctly appears. F: At $196 \mu \mathrm{m}$ depth, the most posterior glomeruli, including G52 and G61, are shown. AN, antennal nerve. Scale bar $=100 \mu \mathrm{m}$ in A.
1986). Mass staining of labial palp receptor neurons in the $H$. virescens female has resulted in heavy labeling of a glomerulus located at a corresponding position (Almaas personal communication). Another relatively large compartment, G34, was located adjacent to the LPO glomerulus, at the dorsomedial side (Figs. 2C-E, 3C,D, 4D, 5D). The unit G61 was also noteworthy in its position posterior to the dorsomedial MGC compartment (Figs. 2E,F, 3D,E, $5 \mathrm{C})$. The smallest glomerulus, G16, was located in a frontolateral part of the antennal lobe (Figs. 2A, 5A) and had an axial thickness of only $16 \mu \mathrm{m}$ (Table 1 ).

Except for two units, all the glomeruli in specimen 2 were identified according to specimen 1 as shown in Figures 2 and 3 . The array of ordinary glomeruli was highly stereotyped and showed only minor differences between individuals, for instance, in the relative position of G48 and G49 (Figs. 2F, 3E,F). The unidentified glomeruli were $\mathrm{nn} 1$, which was located posteromedially in the antennal lobe, surrounded by G51, G55, G54, and G53 (Fig. 3E), and $\mathrm{nn} 2$, which was located posteriorly to G34 (Fig. 3E,F). Two of the glomeruli in the atlas, the smallest, G16 (Fig. 2A), and the medially located, G57 (Fig. 2D), were not found in specimen 2. Thus, the total number of counted glomeruli in the two specimens was the same, 65 . Also by comparing the $3 \mathrm{D}$ reconstruction of specimen 2 (Fig. 4D) with the labelled atlas (Fig. 5A), the conformity of the glomerular pattern was demonstrated.

\section{Reconstructions of the glomeruli in the $H$. virescens male}

The MGC. The MGC of the H. virescens male appeared in an image section of $35 \mu \mathrm{m}$ depth from the most anterior glomerulus, well separated from the array of the ordinary glomeruli (Fig. 4H, Table 2). As shown in Figure 4G, four compartments constitute this particular complex, previously described morphologically and functionally by Vickers et al. (1998) and Berg et al. (1998). The large cumulus is located close to the entrance of the antennal nerve, another large glomerulus dorsomedially of the cumulus, and two smaller compartments ventrally: the ventrolateral and the ventromedial. In the frontally scanned preparations, the four MGC compartments appeared in the following sequence from anterior to posterior: (1) the ventrolateral, (2) the ventromedial (3) the cumulus, and (4) the dorsomedial compartment. The size of the cumulus showed a maximal thickness of $116 \mu \mathrm{m}$ in specimen 1 , whereas the dorsomedial compartment measured $84 \mu \mathrm{m}$. The two ventrally located MGC compartments had the 

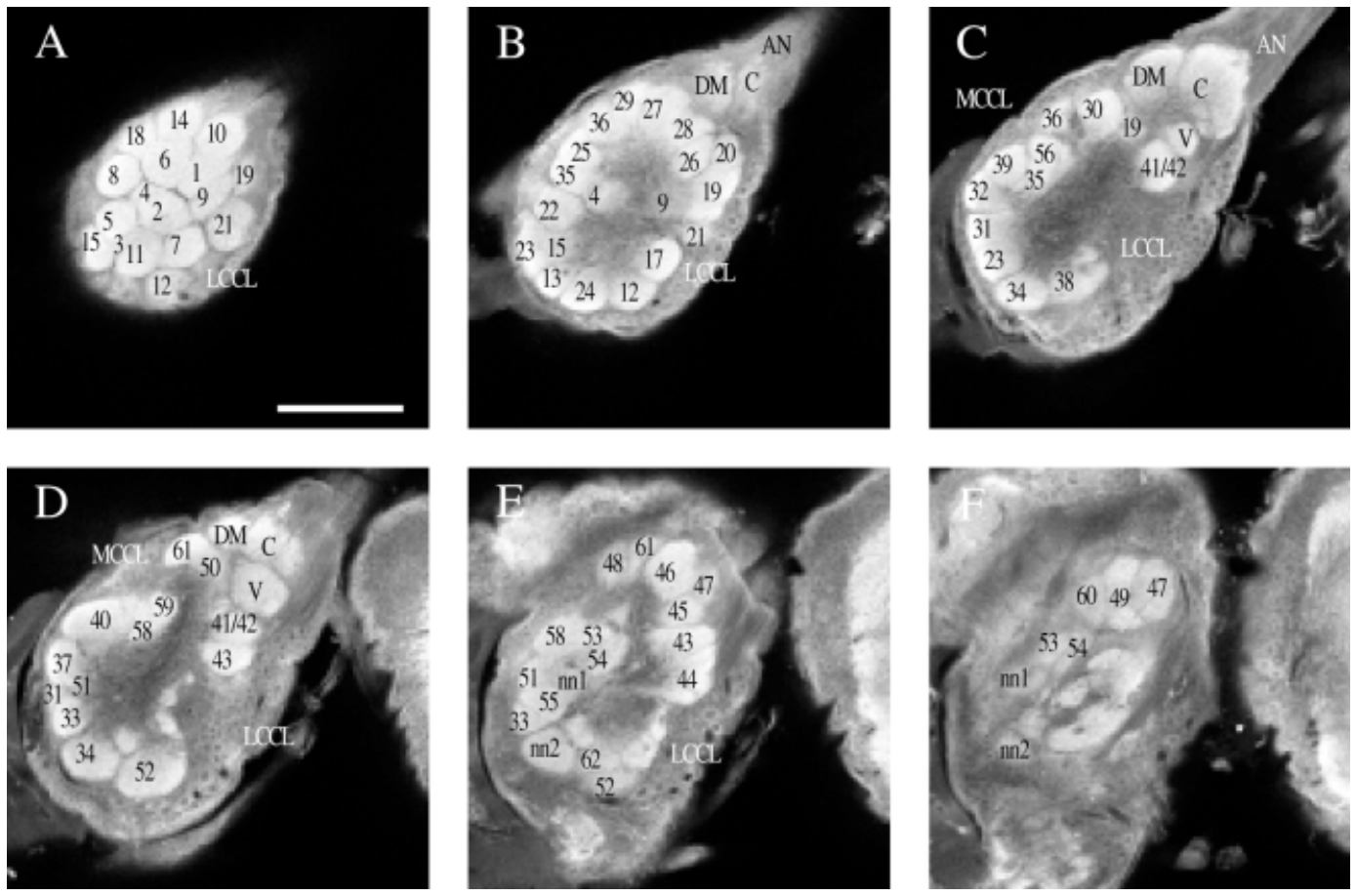

Fig. 3. Helicoverpa assulta male (specimen 2). Images of the antennal lobe in six different planes, showing each of the 63 identified and the two unidentified glomeruli. A: At $43 \mu \mathrm{m}$ depth, the most anterior glomeruli are seen (the glomerulus G16 is not present). B: At $73 \mu \mathrm{m}$ depth, two of the three MGC units [the cumulus (C) and the dorsomedial compartment (DM)] appear, residing outside the array of ordinary glomeruli. C: At $103 \mu \mathrm{m}$ depth, all three MGC compartments $[\mathrm{C}, \mathrm{DM}$, and the ventral $(\mathrm{V})]$ are visible. Both groups of cell bodies are present; the large lateral cell cluster $(\mathrm{LCCl})$ and the smaller medial cell cluster (MCCl). D: At $134 \mu \mathrm{m}$ depth, the LPO glomerulus (G52) can be seen with an adjacent large glomerulus (G34). The G57 is not present. E: At $164 \mu \mathrm{m}$ depth, the two unidentified glomeruli nn1 and nn2 can be seen. F: At $195 \mu \mathrm{m}$ depth, the most posterior glomeruli, including the G53 and G54, are shown. The glomeruli G48, G49, and G60 differ slightly compared with specimen 1, as shown in Figure 2F. AN, antennal nerve. Scale bar $=100 \mu \mathrm{m}$ in A.

TABLE 1. Position and Size of Eight Selected Glomeruli in the Antennal Lobe of the Helicoverpa assulta Male ${ }^{1}$

\begin{tabular}{|c|c|c|c|c|c|c|}
\hline & Labeling & First section $^{2}$ & Last section $^{2}$ & $\begin{array}{c}z \text {-Axis } \operatorname{size}^{3} \\
(\operatorname{sp~} 1 / \operatorname{sp} 2)\end{array}$ & $\begin{array}{c}\operatorname{Max} x y \operatorname{size}^{3} \\
\quad(\operatorname{sp} 1 / \text { sp 2) }\end{array}$ & Volume $^{4}$ \\
\hline MGC: cumulus & Cumulus & $25(50)$ & $71(142)$ & $92 / 85$ & $90 / 100$ & 304.2 \\
\hline MGC: dorsomedial & Dorsomedial & $29(58)$ & $68(136)$ & $78 / 79$ & $65 / 75$ & 135.0 \\
\hline MGC: ventral & Ventral & $33(66)$ & $75(150)$ & $84 / 73$ & $70 / 60$ & 150.0 \\
\hline OG: MGC medial & G61 & $70(140)$ & $98(196)$ & $56 / 52$ & $65 / 50$ & 96.0 \\
\hline OG: LPO & G52 & $44(88)$ & $101(202)$ & $114 / 70$ & $95 / 75$ & 349.2 \\
\hline OG: LPO-medial & G34 & $36(72)$ & $92(184)$ & $112 / 60$ & $75 / 65$ & 250.8 \\
\hline OG: smallest glom. & G16 & $30(60)$ & $38(76)$ & $16 /-$ & $26 /-$ & 6.0 \\
\hline OG: typical & G37 & $55(110)$ & $78(156)$ & $46 / 52$ & $53 / 45$ & 82.2 \\
\hline
\end{tabular}

${ }^{1}$ Three MGC compartments (identified by name), four ordinary glomeruli (OG; labeled by number) that were easily recognized, and one randomly chosen, "typical" OG.

${ }^{2}$ The section numbers refer to the image planes, counted from the anterior to the posterior of the antennal lobe of specimen 1 ; for each glomerulus, the section of first and last appearance is indicated. The position (in $\mu \mathrm{m}$ ) along the $z$-axes is given in parentheses.

${ }^{3}$ Thickness along the $\mathrm{z}$-axis and maximum diameter in the xy-plane, measured in specimen 1 (sp 1 ) and specimen 2 (sp 2 ) is given (in $\mu \mathrm{m}$ ).

${ }^{4}$ The volume of specimen 1 , given by $10^{3} \mu \mathrm{m}^{3}$, is calculated by the "tissue statistics" tool of Amira 2.1 .

size of the ordinary glomeruli, about $45 \mu \mathrm{m}$ (Table 2 ). The maximal thickness measured in each of the four MGC compartments of specimen 2 was slightly smaller for the cumulus and the dorsomedial unit, whereas the two ventral compartments were similar (Table 2).

Ordinary glomeruli. In addition to the four MGC compartments, 62 ordinary glomeruli were counted in the $H$. virescens male. Most of them showed a spherical structure with a diameter of 40-60 $\mu \mathrm{m}$ (Fig. 4E,H). One glomerulus, G54, located adjacent to the dorsomedial MGC compartment, was recognized by its relatively large size. With a maximal diameter of about $80 \mu \mathrm{m}$ in specimen 1 and $65 \mu \mathrm{m}$ in specimen 2 , this particular unit resembled the large MGC compartments rather than the ordinary glomeruli (Table 2). Another glomerulus, G39, was easily recognized as the LPO unit based on its large size and ventral position in the antennal lobe (Fig. 4F,H). Adjacent and medially to the LPO unit was the relatively large glomerulus, G35 (Fig. 4F,H). The smallest glomerulus, G23 (Fig. 4F,H), had a thickness of 28 and $25 \mu \mathrm{m}$ in specimens 1 and 2 , respectively (Table 2 ).

Specimen 2 contained the same number of glomeruli as in the atlas. Among the 66 units, 36 glomeruli were identified. These included glomeruli G1-G23, G25-G28, G31, G35, G39, G54, G62, and the four MGC compartments. Except for G54, all of them are shown in Figure 4F,H. One 

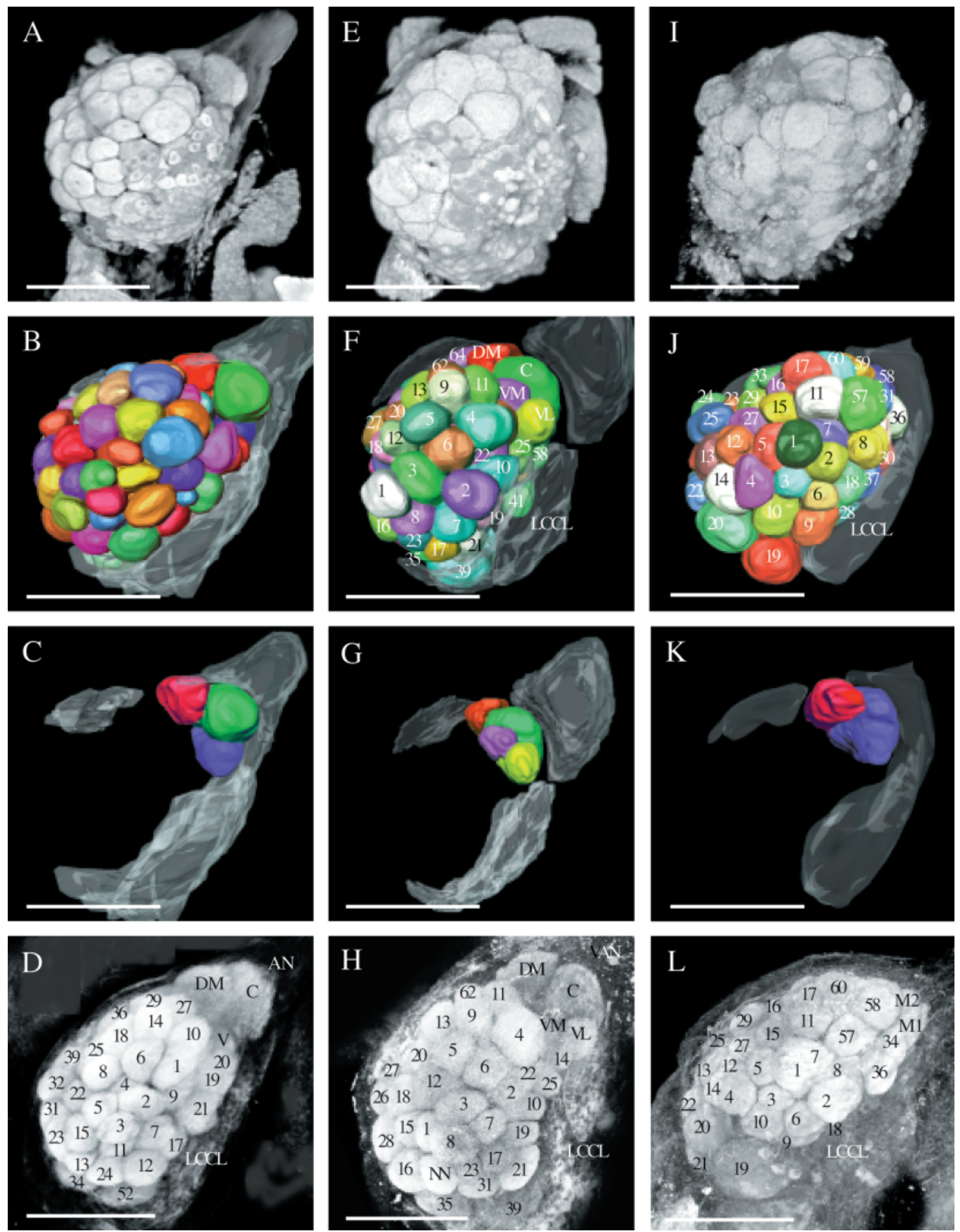

Fig. 4. Three-dimensional reconstructions of the antennal lobe of the Helicoverpa assulta male (left column), the Heliothis virescens male (middle column), and the Heliothis virescens female (right column). A,E,I: Data from the confocal stacks of each preparation (specimen 1), visualized by using the "volume rendering" tool of Amira. $\mathbf{B , F}, \mathbf{J}$ : The digital atlases of each preparation, obtained by using the segmentation tool of Amira. Each glomerulus is given a distinct value, which is visualized as an individual color (The $H$. virescens atlases are labeled, whereas the $H$. assulta atlas is labeled in Fig. 5). C,G,K: Surface views of the male-specific MGCs and the large female glomeruli (LFG) obtained by segmentation. D,H,L: Data from the confocal stacks of each second preparation (specimen 2), visualized by using the "projection" tool of Zeiss LSM 510. The cLFG and the mLFG are named $M 1$ and $M 2$, respectively. Scale bars $=200 \mu \mathrm{m}$. 

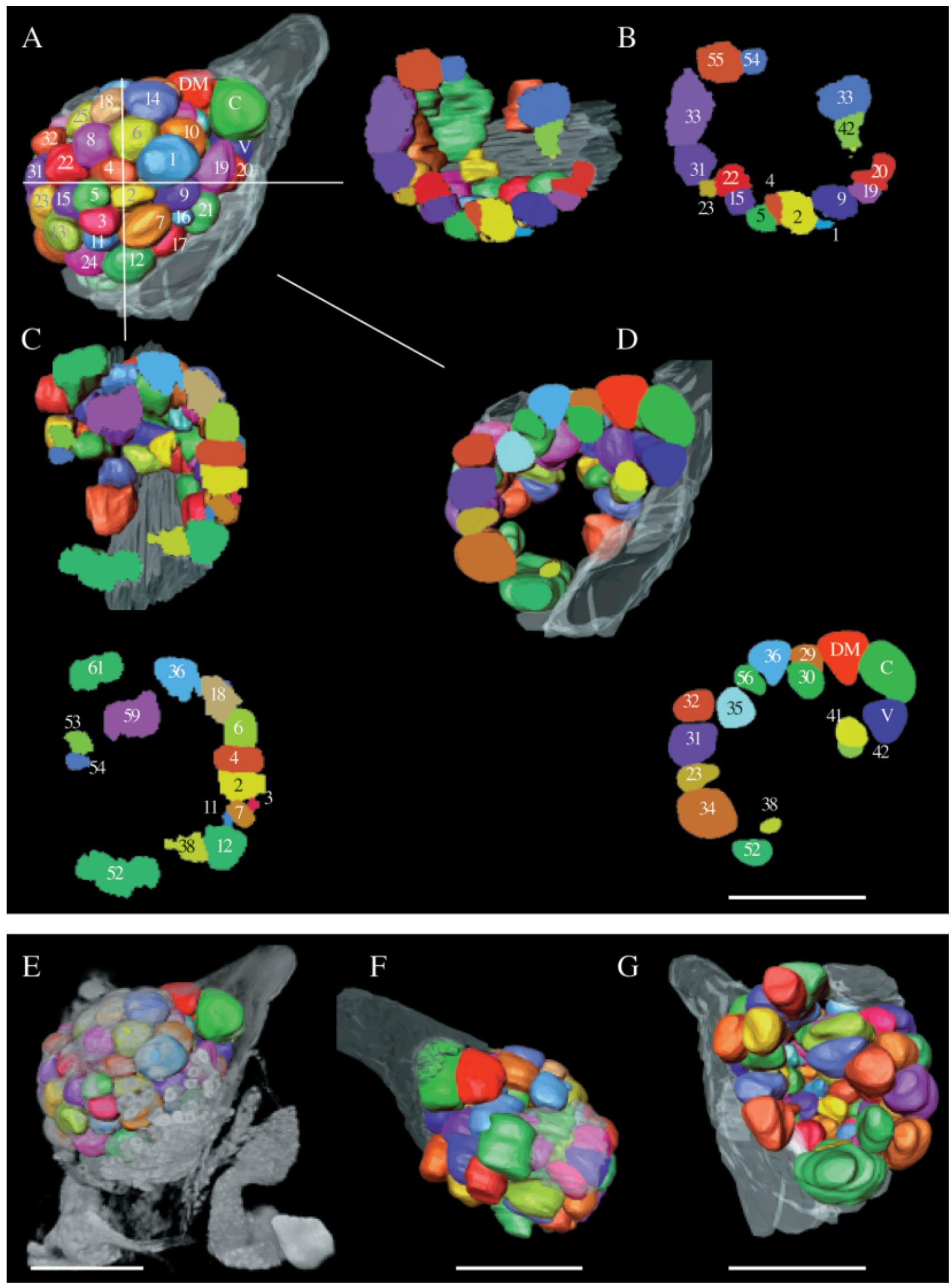
TABLE 2. Position and Size of Nine Selected Glomeruli in the Antennal Lobe of the Heliothis virescens Male ${ }^{1}$

\begin{tabular}{|c|c|c|c|c|c|c|}
\hline & Labeling & First section ${ }^{2}$ & Last section $^{2}$ & $\begin{array}{c}z \text {-Axis size }{ }^{3} \\
(\mathrm{sp} 1 / \mathrm{sp} \mathrm{2})\end{array}$ & $\begin{array}{c}\operatorname{Max}_{x y} \operatorname{size}^{3} \\
(\mathrm{sp} 1 / \mathrm{sp} 2)\end{array}$ & Volume $^{4}$ \\
\hline MGC: cumulus & Cumulus & $15(60)$ & $44(176)$ & $116 / 83$ & $110 / 90$ & 481.2 \\
\hline MGC: dorsomedial & Dorsomedial & $19(76)$ & $40(160)$ & $84 / 71$ & $72 / 65$ & 229.8 \\
\hline MGC: ventrolateral & Ventrolateral & $10(40)$ & $22(88)$ & $48 / 42$ & $55 / 45$ & 75.6 \\
\hline MGC: ventromedial & Ventromedial & $12(48)$ & $22(88)$ & $40 / 37$ & $48 / 40$ & 48.0 \\
\hline OG: MGC medial & G54 & 32 (128) & 47 (188) & $60 / 58$ & $80 / 65$ & 164.0 \\
\hline OG: LPO & G39 & $25(100)$ & $51(204)$ & $104 / 68$ & $93 / 65$ & 389.4 \\
\hline OG: LPO-medial & G35 & $26(104)$ & $42(168)$ & $64 / 55$ & $70 / 60$ & 396.0 \\
\hline OG: smallest glom. & G23 & $9(36)$ & $16(64)$ & $28 / 25$ & $35 / 32$ & 26.4 \\
\hline OG: typical & G5 & $2(8)$ & $13(52)$ & $44 / 34$ & $50 / 50$ & 66.0 \\
\hline
\end{tabular}

${ }^{1}$ Four MGC compartments (identified by name), three ordinary glomeruli (OG; labeled by number) that were easily recognized, the smallest glomerulus, and one randomly chosen, "typical" OG.

${ }^{2}$ The section numbers refer to the image planes, counted from the anterior to the posterior of the antennal lobe of specimen 1 ; for each glomerulus, the section of first and last appearance is indicated. The position (in $\mu \mathrm{m}$ ) along the $z$-axes is given in parentheses.

${ }^{3}$ Thickness along the z-axis and maximum diameter in the xy-plane are given (in $\mu \mathrm{m}$ ), measured in specimen 1 (sp 1) and specimen 2 (sp 2).

${ }^{4}$ The volume of specimen 1 , given by $10^{3} \mu^{3}$, is calculated by the "tissue statistics" tool of Amira 2.1 .

frontally located glomerulus, $\mathrm{NN}$, found in specimen 2 , is not present in the atlas (Fig. $4 \mathrm{H})$.

\section{Reconstructions of the glomeruli in the $H$. virescens female}

Among the 62 glomeruli counted in the $H$. virescens female, two large compartments were found dorsolaterally in the antennal lobe. Their position close to the entrance of the antennal nerve corresponds to the location of the MGC in the male (Fig. 4K). Differently from the MGC, however, which is clearly separated from the ordinary glomeruli, these two compartments form a more integrated part of the shell-like array of ordinary glomeruli. The two units are named the central large female glomerulus (cLFG) and the medial large female glomerulus (mLFG), corresponding to the lateral and the medial large glomeruli of the $M$. sexta female (Rospars and Hildebrand, 2000). The cLFG, situated in the middle of the antennal nerve entrance, had a maximal thickness of $110 \mu \mathrm{m}$ in specimen 1 , whereas the medial compartment measured $102 \mu \mathrm{m}$ (Table 3). The large female glomeruli appeared in a section at $125 \mu \mathrm{m}$ depth from the most anterior glomerulus of the antennal lobe. The position of the two compartments, here appearing much deeper than the MGC of the males, is probably caused by the more ventral orientation of the preparation.

Most of the 60 remaining glomeruli counted in the $H$. virescens female were spheroid or ellipsoid, with a diameter of 40-60 $\mu \mathrm{m}$ (Fig. 4I,J,L). Also in the female the large LPO glomerulus, G19, located ventrally in the antennal

Fig. 5. Helicoverpa assulta. A: Digital three-dimensional atlas of the antennal lobe with labeled glomeruli. B: Coronal section through the 3D reconstruction as indicated by the horizontal line. At right, one section is shown with labeled glomeruli. At left, the same section is shown implanted in the ventral part of the $3 \mathrm{D}$ reconstruction. C: Sagittal section through the $3 \mathrm{D}$ reconstruction as indicated by the vertical line. Below, one section is shown with numbered glomeruli. At the top, the same section is shown implanted in the medial part of the 3D reconstruction. D: Frontal section through the $3 \mathrm{D}$ reconstruction. At right, one section is shown with labeled glomeruli (100 $\mu \mathrm{m}$ depth; same section as shown in Fig. 2C). At left, the same section is shown implanted in the posterior part of the 3D reconstruction. E: Three-dimensional reconstruction of the deutocerebrum, shown by the combination of two visualization techniques; the total assembly of glomeruli, reconstructed by use of the segmentation tool of Amira, is implanted in the $3 \mathrm{D}$ reconstruction of the remaining brain structures obtained by the "volume rendering" tool. F: Dorsoposterior view of the digital atlas. G: Posterior view of digital atlas. Scale bars $=200 \mu \mathrm{m}$ in D-G. lobe, was recognized (Fig. 4J,L). A large glomerulus located medially to the LPO compartment was the G20 unit. The smallest glomerulus, G3, measured $40 \mu \mathrm{m}$ in specimen 1 and 35 in specimen 2 (Table 3). Altogether, specimen 2 contained 65 glomeruli, i.e., three more than the atlas. Thirty-two of them were identified according to the atlas and included, in addition to the two LFG compartments, glomeruli G1-G22, G25, G27, G29, G34, G36, G57, G58, and G60. All of them are shown in Figure 4L. Although the sizes of the glomeruli G19, G20, and G3 were similar in the two specimens, the two LFG compartments (cLFG and mLFG) were considerably smaller in specimen 2, measuring 72 and $68 \mu \mathrm{m}$, respectively (Table 3).

\section{Reconstructions of the lateral and the medial cell cluster of the antennal lobe}

In all six preparations, the two main cell clusters of the antennal lobe were easily recognized as densely packed somata located at the lobe periphery (Figs. 2, 3). The largest, the lateral cell cluster, appeared in one of the first image planes, continuing in a ventrolateral position throughout the antennal lobe. This large assembly of cell bodies sends its neurites in a bundle penetrating the outer shell of glomeruli. The considerably smaller medial cell cluster appears more posteriorly in the antennal lobe (Figs. 2C, 3C, Table 4).

\section{DISCUSSION}

". . . There is nothing casual in its [the universal organism] life but a single melody and order ..." (Armstrong, 1984). This quotation of Plotinus comes to mind when considering the appearance of the discrete neuropil structures forming the glomeruli of the primary olfactory center of most animals. Indeed, the well-organized system of distinct glomeruli in the antennal lobe of the heliothine moth seems to reflect the truth of these words. The use of an antibody recognizing synaptic structures resulted in distinct staining of the glomeruli in the two moth species investigated here. Thus, the glomerular units are visualized as condensed collections of synapses, according to the definition of glomeruli as sites of increased synaptic density (Shepherd, 1974). The remaining area of the deutocerebrum, the antennal mechanosensory and motor center, was also strongly labeled but lacked the glomerular organization. This is in accordance with previous anatomical studies of the two main deutocerebral divisions (Rospars, 1988; Homberg et al., 1989; Kloppenburg et al., 
TABLE 3. Position and Size of Six Selected Glomeruli in the Antennal Lobe of the Heliothis virescens Female ${ }^{1}$

\begin{tabular}{|c|c|c|c|c|c|c|}
\hline & Labeling & First section ${ }^{2}$ & Last section $^{2}$ & $\begin{array}{c}z \text {-Axis size } \\
(\operatorname{sp~} 1 / \operatorname{sp~} 2)\end{array}$ & $\begin{array}{c}\text { Max xy size } \\
\quad(\operatorname{sp~} 1 / \text { sp } 2)\end{array}$ & Volume $^{4}$ \\
\hline OG: LFG central & CLFG (M1) & 64 (128) & 119 (238) & $110 / 72$ & $112 / 65$ & 560.4 \\
\hline OG: LFG medial & MLFG (M2) & $60(120)$ & $111(222)$ & $102 / 68$ & $75 / 63$ & 267.0 \\
\hline OG: LPO & G19 & $36(72)$ & $76(152)$ & $80 / 67$ & $80 / 78$ & 283.8 \\
\hline OG: LPO medial & G20 & $28(56)$ & $54(108)$ & $52 / 52$ & $70 / 60$ & 157.2 \\
\hline OG: smallest & G3 & $9(18)$ & $29(58)$ & $40 / 35$ & $40 / 40$ & 34.2 \\
\hline OG: typical & G17 & $22(44)$ & $44(88)$ & $44 / 49$ & $60 / 50$ & 96.0 \\
\hline
\end{tabular}

${ }^{1}$ Two LFG compartments (labeled by name), two glomeruli (labeled by number) that were easily recognized, the smallest glomerulus, and one randomly chosen "typical" ordinary glomerulus.

${ }^{2}$ The section numbers refer to the image planes counted from the anterior to the posterior of the antennal lobe of specimen 1 ; for each glomerulus, the section of first and last appearance is indicated. The position (in $\mu \mathrm{m}$ ) along the $z$-axes is given in parentheses.

${ }^{3}$ Thickness along the $z$-axis and maximum diameter in the xy-plane are given (in $\mu \mathrm{m}$ ), measured in specimen 1 (sp 1) and specimen 2 (sp 2).

${ }^{4}$ The volume of specimen 1 , given by $10^{3} \mu \mathrm{m}^{3}$, is calculated by the "tissue statistics" tool of Amira 2.1 .

TABLE 4. Location and Size of the Main Cell Clusters of the Antennal Lobe in the Heliothine Species Helicoverpa assulta (Male) and Heliothis virescens (Male and Female)

\begin{tabular}{|c|c|c|c|c|c|}
\hline & $\begin{array}{l}\text { Cell } \\
\text { cluster }\end{array}$ & First section $^{1}$ & Last section $^{1}$ & $\begin{array}{c}z \text {-Axis } \\
\operatorname{size}(\mu \mathrm{m})\end{array}$ & $\begin{array}{c}\operatorname{Max} x y \text { size } \\
(\mu \mathrm{m})\end{array}$ \\
\hline \multirow[t]{2}{*}{ Helicoverpa assulta male } & $\mathrm{LCCl}$ & $1(2)$ & 74 (148) & 146 & 245 \\
\hline & $\mathrm{MCCl}$ & 47 (94) & $95(190)$ & 96 & 150 \\
\hline \multirow{2}{*}{ Heliothis virescens male } & $\mathrm{LCCl}$ & $2(8)$ & $40(160)$ & 152 & 245 \\
\hline & $\mathrm{MCCl}$ & $20(40)$ & 46 (184) & 104 & 163 \\
\hline \multirow[t]{2}{*}{ Heliothis virescens female } & $\mathrm{LCCl}$ & $30(60)$ & $85(170)$ & 110 & 250 \\
\hline & $\mathrm{MCCl}$ & $73(146)$ & $120(240)$ & 94 & 125 \\
\hline
\end{tabular}

${ }^{1}$ The section numbers refer to the image planes, counted from anterior to posterior in the antennal lobe of specimen 1; for each cell cluster, the section of first and last appearance is indicated. The position (in $\mu \mathrm{m}$ ) along the z-axes is given in parentheses. LCCl, lateral cell cluster; MCCl, medial cell cluster.

1997). The staining of the cell clusters, in addition to the somewhat weaker labeling of the antennal nerve, was beneficial, in that these structures represent reliable landmarks for orientation. Labeling of cell somata and axons is probably due to the presence of synapsin intracellularly in these structures. It has been shown in vertebrates that both structures are stained by high concentrations of synapsin antibodies, whereas lower concentrations stain only the axon terminals.

The small heliothine brain is advantageous for confocal microscopy; the entire antennal lobe, as well as the whole brain, can be visualized as a single stack of sequential optical images. The efficiency of the staining technique and the resolution of the images, showing an interglomerular space of $2 \mu \mathrm{m}$ in some sections (Fig. 2), made it possible to outline the borders of the individual glomeruli. A similar technique, using a neuropil-specific antibody, has been used for the computerized map of the Drosophila antennal lobe (Laissue et al., 1999), whereas the honeybee atlas was based on a different staining technique, tracing the sensory axons with a specific dye (Galizia et al., 1999). Labeling of the primary axons, which was also attempted in the present study, was insufficient for segmentation. A combination of different staining techniques may further improve the visualization of the brain structures in heliothine moths. It would be of particular interest to resolve the antennal tracts in order to determine their innervation of the glomeruli. In the honeybee, the antennal nerve splits into four tracts that innervate the antennal lobe. These tracts have been used as the basis for the nomenclature of the glomeruli (Flanagan and Mercer, 1988). For the moth, such a system is not yet available. A list of five antennal tracts has been described for $M$. sexta, but without mapping their innervation pattern to the glomeruli (Rospars and Hildebrand, 1992). The terminology used in the fly atlas is based on the exact position of the 43 glomeruli, i.e., their position according to all three axes $(x, y, z$; Stocker et al., 1990; Laissue et al., 1999). Because of the relatively large number of units and the lack of visualized tracts, we found that it was appropriate in this study of heliothine moths to use the more incidental system of labeling the glomeruli by numbers according to their position along the $z$-axis.

Each of the three digital atlases presented here is based on the reconstruction of one antennal lobe. Analysis carried out by comparing a second specimen with the original indicates only small variations of the glomerular pattern within each of the three types. The number of counted glomeruli was identical in the two male individuals of $H$. assulta (65) as well as of $H$. virescens (66) and differed only by three in the two female individuals of $H$. virescens (62/65). A relatively consistent glomerular pattern was also found, showing only minor differences concerning one or two glomeruli in each type. Interestingly, the smallest glomerulus, G16, in the $H$. assulta male atlas was not found in specimen 2 . The results presented here are in accordance with previous findings showing that the number and position of the glomeruli are relatively invariant across individuals of the insect species (Rospars and Hildebrand, 1992, 2000; Galizia et al., 1999). Whereas small variations in the array of the numerous ordinary glomeruli were present, the number, position, and shape of the MGC compartments were strictly constant within the species, which is also consistent with previous findings in heliothine moths (cf. Mustaparta, 1997). It is interesting that the number of counted glomeruli in the six specimens is relatively constant, 62, 65, 65, 65, 66, and 66 . This is in the range of the number found in other moth species, for instance, $M$. sexta, with 63 glomeruli (Rospars and Hildebrand, 2000), and Mamestra brassicae, with 67 male and 68 female glomeruli (Rospars, 1983).

Some glomeruli can be considered as homologous in different species. This particularly applies to the two MGC compartments, the cumulus and the dorsomedial unit, in males of $H$. virescens and $H$. assulta as well as of other heliothine species (Christensen et al., 1991; Vickers et al., 
1998; Berg et al., 1998; T.J. Almaas, unpublished observations). In addition to the anatomical correspondence, the functional aspects show interesting similarities. The cumulus, located close to the entrance of the antennal nerve, always receives input about the major pheromone component (Berg et al., 1998). It is the same chemical, cis-11-hexadecenal, in all heliothine species studied so far (Arn et al., 1992), except for H. assulta, which uses cis-9hexadecenal (Cork et al., 1992). The dorsomedial compartment always receives input about another compound, cis9 -tetradecenal. However, in this case, the message differs, being a prerequisite for pheromone attraction in $H$. virescens (Roelofs et al., 1974) and an interruptant of the attraction in $H$. assulta (Boo et al., 1995), Helicoverpa zea (Shaver et al., 1982), and Helicoverpa armigera (Kehat and Dunkelblum, 1990). Thus, the dorsomedial compartment has the same location and receives input information about the same chemical in all heliothine species investigated but differs with respect to the behavior it elicits when excited by an olfactory stimulus.

Homologous glomerular structures may also be present in the heliothine females. In the one female investigated here, of $H$. virescens, two enlarged compartments were shown at the entrance of the antennal nerve, in a position corresponding to the male-specific MGC, the largest residing centrally at the antennal nerve entrance and a somewhat smaller unit in the dorsomedial position. Large female glomeruli have previously been described for the silk moth Bombyx mori (Koontz and Schneider, 1987) as well as for M. sexta (Rössler et al., 1998). It has recently been reported that one of the two units identified in the latter mediates information specifically about the plant volatile linalool (King et al., 2000). The function of the large female glomeruli in $H$. virescens is not yet clarified.

Among the ordinary glomeruli, the LPO compartment was particularly easy to recognize in all six specimens $[H$. assulta male: G52 (Figs. 2, 3, 4D, Table 1), $H$. virescens male: G39 (Fig. 4F,H, Table 2), $H$. virescens female: G19 (Fig. 4J,L, Table 3)]. This is the largest ordinary glomerulus, residing most ventrally in the antennal lobe. Another large glomerulus, found in all specimens, was located adjacent to the LPO unit, on the medial side $[H$. assulta male: G34 (Figs. 2, 3, 4D, Table 1), H. virescens male: G35 (Fig. 4F,H, Table 2), H. virescens female: G20 (Fig. 4J,L, Table 3)]. A glomerulus situated adjacent to the dorsomedial MGC compartment, in the posterior part of the antennal lobe, was found in the four male preparations [H. assulta male: G61 (Figs. 2, 3, Table 1), $H$. virescens male: G54 (Table 2)]. Particularly in $H$. virescens this unit, G54, was conspicuous by its large size. Interestingly, a large glomerulus occupying a location next to the MGC has also been identified in both sexes of $M$. sexta. It is noteworthy that the number of ordinary glomeruli in the male and female of $H$. virescens was similar (62/62 and $62 / 65$, respectively). According to intersexual homology, a recent study of the antennal lobe anatomy of adult male and female $M$. sexta has shown that 60 ordinary glomeruli are isomorphic in the two sexes, whereas three are dimorphic (Rospars and Hildebrand, 2000).

The manner in which the glomeruli in general operate as functional units is not yet fully understood for any organism. However, results from various studies indicate that they may represent a physical basis for a functional map of odor qualities. The use of transgenic $D$. melanogaster has recently shown that receptor neurons expressing one type of receptor molecules project exclusively to one (or sometimes two) homologous glomerulus in each antennal lobe (Gao et al., 2000; Vosshall et al., 2000). These and other data from molecular biological studies (Ressler et al., 1994; Vassar et al., 1994; Mombaerts et al., 1996; Wang et al., 1998) are in accordance with the results obtained for the pheromone system in the heliothine moth, showing that neurons of one functional type project to one MGC unit (Hansson et al., 1992; Berg et al., 1998). In addition, data from calcium imaging of the antennal lobe of the honeybee have demonstrated that different odor qualities induce a specific pattern of glomerular activation (Joerges et al., 1997; Galizia and Menzel, 2000). By using the same imaging technique, a pattern of glomerular activation of the MGC that corresponds to the functional organization demonstrated by tracing of receptor neurons has recently been shown for $H$. virescens (Galizia et al., 2000). Also in this case a specific activation pattern of the ordinary glomeruli was obtained by stimulation with different plant odors. To study in detail which of the 60-62 ordinary glomeruli are activated by the various biologically relevant plant odors in the antennal lobe of heliothine moths, it is necessary to have an atlas of the glomeruli in these species.

\section{ACKNOWLEDGMENTS}

We thank Randolf Menzel for his unlimited and creative support. This study has been supported in varying parts by The Norwegian Research Council (grant 125821/410 to B.G.B. and grant $133958 / 420$ to H.M.), by the German Research Ministry BMBF (grant 0310961 to R.M.), and by the Volkswagenstiftung (grant 175/399 to C.G.G.).

\section{LITERATURE CITED}

Armstrong AH. 1984. Plotinus. Cambridge, MA: Harvard University Press. Arn H, Toth M, Priesner E. 1992. List of sex pheromones and related attractants. International Organization for Biological Control, West Palearctic Regional Section.

Berg BG, Almaas TJ, Bjaalie JG, Mustaparta H. 1998. The macroglomerular complex of the antennal lobe in the tobacco budworm moth $\mathrm{He}$ liothis virescens: specified subdivision in four compartments according to information about biologically significant compounds. J Comp Physiol A183:669-682.

Berg BG, Almaas TJ, Mustaparta H. 1999. The macroglomerular complex in two related species of moths: specified subdivision according to input information. Chem Senses 24:566.

Boeckh J, Tolbert LJ. 1993. Synaptic organization and development of the antennal lobe in insects. Microsc Res Techn 24:260-280.

Boo KS, Park KC, Hall DR, Cork A, Berg BG, Mustaparta H. 1995. (Z)-9-tetradecenal: a potent inhibitor of pheromone-mediated communication in the oriental tobacco budworm moth, Helicoverpa assulta. J Comp Physiol A177:695-699.

Christensen TA, Mustaparta H, Hildebrand JG. 1991. Chemical communication in heliothine moths II. Central processing of intra- and interspecific olfactory messages in the male corn earworm moth Helicoverpa zea. J Comp Physiol A180:523-536.

Christensen TA, Mustaparta H, Hildebrand JG. 1995. Chemical communication in heliothine moths VI. Parallel pathways for information processing in the macroglomerular complex of the male tobacco budworm moth Heliothis virescens. J Comp Physiol A177:545-557.

Cork A, Boo KS, Dunkelblum E, Hall DR, Ree-Rajunga K, Kehat M, Kong Jie E, Park KC, Tepkidagarn P, Liu Xun. 1992. Female sex pheromone of oriental tobacco budworm, Helicoverpa assulta (Guenée) (Lepidoptera: Noctuidae): identification and field testing. J Chem Ecol 18:403-418.

Flanagan D, Mercer AR. 1988. An atlas and 3-D reconstruction of the antennal lobes in the worker honey bee, Apis mellifera L. (Hymenoptera: Apidae). Int J Insect Morphol Embryol 18:145-159.

Galizia CG, Menzel R. 2000. Odour perception in honeybees: coding information in glomerular patterns. Curr Opin Neurobiol 10:504-510. 
Galizia CG, McIlwrath SL, Menzel R. 1999. A digital three-dimensional atlas of the honeybee antennal lobe based on optical sections acquired by confocal microscopy. Cell Tissue Res 295:383-394.

Galizia CG, Sachse S, Mustaparta H. 2000. Calcium responses to stimulation with pheromones and plant odours in the antennal lobe of the male and female moth Heliothis virescens. J Comp Physiol A186:10491063.

Gao Q, Yuan B, Chess A. 2000. Convergent projections of Drosophila olfactory neurons to specific glomeruli in the antennal lobe. Nat Neurosci 3:780-785.

Hansson B, Christensen TA, Hildebrand JG. 1991. Functionally distinct subdivision of the macroglomerular complex in the antennal lobe of the male sphinx moth Manduca sexta. J Comp Neurol 312:264-278.

Hansson BS, Ljungberg H, Hallberg E, Løfstedt C. 1992. Functional specialisation of olfactory glomeruli in a moth. Science 256:1313-1315.

Hansson BS, Almaas TJ, Anton S. 1995. Chemical communication in heliothine moths V. Antennal lobe projection patterns of pheromonedetecting olfactory receptor neurons in the male Heliothis virescens (Lepidoptera: Noctuidae). J Comp Physiol A177:535-543.

Homberg U, Montague RA, Hildebrand JG. 1988. Anatomy of antennocerebral pathways in the brain of the sphinx moth Manduca sexta. Cell Tissue Res 254:255-281.

Homberg U, Christensen TA, Hildebrand JG. 1989. Structure and function of the deutocerebrum in insects. Annu Rev Entomol 34:477-501.

Joerges J, Kuettner A, Galizia CG, Menzel R. 1997. Representations of odours and odour mixtures visualized in the honeybee brain. Nature $387: 285-288$

Kehat M, Dunkelblum E. 1990. Behavioural responses of male Heliothis armigera (Lepidoptera: Noctuidae) moths in a flight tunnel to combinations of components identified from female sex pheromone glands. J Insect Behavior 3:75-83.

Kent KS, Harrow ID, Quatararo P, Hildebrand JG. 1986. An accessory olfactory pathway in Lepidoptera: the labial pit organ and its central projections in Manduca sexta and certain other sphinx moths and silk moths. Cell Tissue Res 245:237-245.

King JR, Christensen TA, Hildebrand JG. 2000. Response characteristics of an identified, sexually dimorphic olfactory glomerulus. J Neurosci 20:2391-2399.

Klagges BRE, Heimbeck G, Godenschwege TA, Hofbauer A, Pflugfelder GO, Reifegerste R, Reisch D, Schaupp M, Buchner E. 1996. Invertebrate synapsins: a single gene codes for several isoforms in Drosophila. J Neurosci 16:3154-3165.

Kloppenburg P, Camazine SM, Sun XJ, Randolph P, Hildebrand JG. 1997. Organization of the antennal motor system in the sphix moth Manduca sexta. Cell Tissue Res 287:425-433.

Koontz MA, Schneider D. 1987. Sexual dimorphism in neuronal projections from the antennae of silk moths (Bombyx mori, Antheraea polyphemus) and the gypsy moth (Lymantria dispar). Cell Tissue Res 249:39-50.

Laissue PP, Reiter C, Hiesinger PR, Halter S, Fischbach KF, Stocker RF. 1999. Three-dimensional reconstruction of the antennal lobe in Drosophila melanogaster. J Comp Neurol 405:543-552.

Mombaerts P, Wang F, Dulac C, Chao SK, Nemes A, Mendelsohn M,
Edmondson J, Axel R. 1996. Visualizing an olfactory sensory map. Cell 87:675-686.

Mustaparta H. 1997. Olfactory coding mechanisms. In: Cardé RT, Minks $\mathrm{AK}$, editors. Insect pheromone research. New directions. New York: Chapman and Hall. p 144-163.

Reichmuth C, Becker S, Benz M, Reisch D, Heimbeck G, Hofbauer A, Klagges BRE, Pflugfelder GO, Buchner E. 1995. The sap47 gene of Drosophila melanogaster codes for a novel conserved neuronal protein associated with synaptic terminals. Mol Brain Res 32:45-54.

Ressler KJ, Sulliva SL, Buck LB. 1994. Information coding in the olfactory system: evidence for a stereotyped and highly organized epitope map in the olfactory bulb. Cell 79:1245-1255.

Roelofs WL, Hill AS, Cardé RT, Baker TC. 1974. Two sex pheromone components of the tobacco budworm moth Heliothis virescens. Life Sci 14:1555-1562.

Rospars JP. 1983. Invariance and sex-specific variations of the glomerular organization in the antennal lobes of a moth, Mamestra brassicae, and a butterfly, Pieris brassicae. J Comp Neurol 220:80-96.

Rospars JP. 1988. Structure and development of the insect antennodeutocerebral system. Int J Insect Morphol Embryol 17:243-294.

Rospars JP, Hildebrand JG. 1992. Anatomical identification of glomeruli in the antennal lobes of the male sphinx moth Manduca sexta. Cell Tissue Res 270:205-227.

Rospars JP, Hildebrand JG. 2000. Sexually dimorphic and isomorphic glomeruli in the antennal lobes of the sphinx moth Manduca sexta. Chem Senses 25:119-129.

Rössler W, Tolbert L, Hildebrand JG. 1998. Early formation of sexually dimorphic glomeruli in the developing olfactory lobe of the brain of the moth Manduca sexta. J Comp Neurol 396:415-428.

Røstelien T, Borg-Karlson A-K, Fäldt J, Jacobsson U, Mustaparta H. 2000. The plant sequiterpene Germacrene $\mathrm{D}$ specifically activates a major type of antennal receptor neuron of the tobacco budworm moth $\mathrm{He}$ liothis virescens. Chem Senses 25:141-148.

Shaver TN, Lopez JD Jr, Hartstack AW Jr. 1982. Effects of pheromone components and their degradation products on the responses of $\mathrm{He}$ liothis spp. to traps. J Chem Ecol 8:755-762.

Shepherd GM. 1974. The synaptic organization of the brain. An introduction. New York: Oxford University Press.

Stocker RF, Leinhard MC, Borst A, Fischbach KF. 1990. Neuronal architecture of the antennal lobe in D. melanogaster. Cell Tissue Res 262:9-34.

Vassar R, Chao SK, Sitcheran R, Nuñes JM, Vosshall LB, Axel R. 1994. Topographic organization of sensory projections to the olfactory bulb. Cell 79:981-991.

Vickers NJ, Christensen TJ, Hildebrand JG. 1998. Combinatorial odor discrimination in the brain: attractive and antagonist odor blends are represented in distinct combinations of uniquely identifiable glomeruli. J Comp Neurol 400:35-56.

Vosshall LB, Wong AM, Axel R. 2000. An olfactory sensory map in the fly brain. Cell 102:147-159.

Wang F, Nemes A, Mendelsohn M, Axel R. 1998. Odorant receptors govern the formation of a precise topographic map. Cell 93:47-60.

APPENDIX. Nomenclature of Individual Glomeruli (The Value of Each Voxel Gives the Identity of the Glomerulus)

\begin{tabular}{lcc}
\hline & Helicoverpa assulta, male & Heliothis virescens, male \\
\hline Ordinary glomeruli & G1-G62 as 1-62 & G1-G62 as 1-62 \\
MGC/LFG & Cumulus: 101 & Cumulus: 101 \\
& Dorsomedial: 102 & Dorsomedial: 102 \\
& Ventral: 103 & Ventrolateral: 104 \\
Medial cell cluster & & Ventromedial: 105 \\
Lateral cell cluster & 201 & 201 \\
Antennal nerve & 202 & 202 \\
mLFG: 107 & 203 \\
\hline
\end{tabular}

Format of the atlas on the internet: http://www.neurobiologie.fu-berlin.de/mothalatlas/ or http://kzoo.chembio.ntnu.no/mothAL/. Each atlas is a volumetric representation of a right antennal lobe. It consists of a series of sections. In each section, the position belonging to a particular glomerulus is identified by its color code (or gray-level value). By using any software capable of 3D graphics, it is possible to reslice and/or rotate the atlas. Most software packages have a tool giving the color-table value of a chosen voxel (= pixel in three dimensions). This value reveals the identity of the glomerulus, following the key given in the Appendix.

On the web site, examples of the atlas as well as the raw confocal data can be seen as Quicktime movies. To work with the atlas, it is best to download the data onto a local computer. The atlas can then be used with one's own software. Basically, each atlas consists of a series of $z$-axis sections, where each glomerulus is identified by a value (color). Because not all software can read all data formats, we provide the identical data in different formats. It is not necessary to download all the material. Rather, find out what your software can read, and then download that particular format.

We have included, for each species, the following formats: three-dimensional TIFF file, compressed and not compressed; three-dimensional GIF file; Amira-mesh file (readable only with the amira software); individual TIFF files for each layer; individual GIF files for each layer; individual BMP files for each layer. The atlas will soon be accessible also from other link. 